\title{
Dolus y Duellum: Pérdida, dolor y duelo en la consulta
}

\author{
doi: 10.33264/rpa.202001-13 \\ Ricardo L. Guzmán Sanza \\ Escuela de Psicología \\ Facultad de Humanidades y Ciencias Sociales
}

\section{Resumen}

El presente trabajo se articula en torno al concepto de duelo que emerge en el espacio terapéutico, producto de la pérdida y el dolor asociado a ella. A partir del análisis de casos en la praxis de la consulta, se conceptualiza el duelo abarcando aspectos y situaciones asociadas a las diferentes pérdidas que se producen en variados aspectos del acontecer. Así, desde una mirada más amplia, se muestra como el proceso de la pérdida y el duelo subsecuente constituye una propia realidad que más que obedecer a etapas predeterminadas, ofrece un espacio de revisión de nuestro mundo de significados y de la apertura a experimentar el dolor, desde nuestra propia realidad. En la primera parte se abordan aspectos teóricos y clínicos, especialmente aquellos que emergen del DSM IV, DSM V y CIE-10, para después de efectuar una presentación de diferentes casos donde la pérdida se asocia a diferentes vivencias, se construyen reflexiones asociadas al duelo, y a los roles del paciente y del terapeuta.

Palabras claves: duelo, dolor, pérdida, terapia

\section{Abstract}

The present paper is articulated around the grief concept which emerges from the therapeutical space, as the result of loss and the associated pain. From the analysis of cases in the practice of the consultation, grief is conceptualized covering aspects and situations associated with different losses that occur in various aspect of the event. Thus, from a broader view, it is shown how the process of loss and the subsequent mourning constitutes a reality that, rather than obeying to predeterminate stages, offers a space for reviewing our world of meanings and the openness to experiment pain, from our own reality. The first part embraces theoretical and clinical aspects, especially those that emerge from DSM IV, DSM V and $\mathrm{CIE}-10$, so that after making a presentation of different cases where loss is associated to different experiences, reflexions are built regarding grief and to the role of patient and therapist.

Keywords: grief, pain, loss, therapy 
Puedo escribir los versos más tristes esta noche

Pensar que no la tengo. Sentir que la he perdido

\section{Introducción}

Los versos del Poema 20 del Poeta y Premio Nobel de Literatura chileno Pablo Neruda (1904-1973), sirven como guía para comprender el sentido que tiene la pérdida. Una primera mirada nos acerca a entender que es algo que teníamos y que hemos perdido, donde posiblemente en la mayoría de los casos, lo perdido en cualquiera de sus formas no estará más con nosotros.

Una reflexión más profunda nos sitúa en el hecho que en el desarrollo del ciclo de vida, se experimentan en forma continua pérdidas las cuales son vivenciadas en forma muy disímiles, sean por ejemplo originadas en oportunidades laborales, familiares mediante la destrucción del hogar, cosas de las cuales las personas deben desprenderse, amigos que ya no se verán producto de ausencias o rompimiento de afectos, cosas materiales como ser pérdidas de activos, financieras u operacionales y también aquellas que consideramos propias como la salud, las capacidades físicas, las capacidades cognitivas, las capacidades íntimas, la juventud, nuestros amores, nuestros seres queridos, generando situaciones que impactan nuestros significados más personales como la fe y la relación con nuestro medio, haciéndonos sentir confusos e inseguros, adentrándonos a tientas por los caminos inseguros que ha generado la pérdida (Bowly, 1993). El elemento común a todas estas pérdidas es el duelo (del latín dolus, dolor, aflicción) que emerge como una reacción natural que no admite diferencias, y que compromete aspectos físicos, sociales y psicológicos (Meza et al, 2008). Resulta interesante señalar que duelo acepta otra significación, la cual también proveniente del latín duellum que reconoce la acepción como "combate" o "pelea entre dos a consecuencia de un reto o desafío". (Barreto \& soler,2007; RAE, 2001).

A partir de las acepciones señaladas y de su significado implícito, puede concebirse el duelo como un resultante de un proceso de pérdida que se encuentra siempre presente en nuestro acontecer. Existen pérdidas que son universalmente aceptadas y definidas claramente en lo social, como puede ser la pérdida de la salud, la pérdida producto del fallecimiento de un ser querido, y otras que son claramente identificables pues socialmente son aceptadas y normalizadas como tal. Pero que sucede con aquellas pérdidas que no resultan tan nítidas para la sociedad y que solo en la mayoría de los casos impacta a la persona en forma individual, como la pérdida 
de una oportunidad laboral, la pérdida producida por la ruptura de un pololeo, la pérdida de confianza en el prójimo, la pérdida del sustento diario, solo por nombrar algunas.

En los casos señalados, el dolor que produce la pérdida puede verse aumentado debido a la incomprensión, falta de atención o apoyo que el doliente recibe de su medio. La pérdida no es solo el sentimiento que se produce por la muerte, si bien es la más importante, sino que abarca el conjunto de experiencias significativas de separación o privación de personas u objetos con las que se ha establecido un vínculo o un lazo. Esta privación que puede ser total o parcial con lo que nos resulta significativo, establece la necesidad de contar con un proceso de elaboración de la relación con lo perdido, que es lo que llamamos duelo. Conviene reflexionar en este punto en torno a que la pérdida en nuestro diario acontecer nos acompaña en todas nuestras acciones desde el momento que nacemos donde perdemos la seguridad del vientre materno, cuando nos separamos de nuestro soporte para dar los primeros pasos, cuando dejamos atrás eventos de nuestra vida como cambio de lugar, de colegio, ingreso a los distintos niveles educacionales donde como en una película algunos amigos aparecen y otros desaparecen, la pérdida de relaciones afectivas, las producidas por engaños, y muchas otras que pudieran identificarse.

Las pérdidas son múltiples y se van sucediendo una tras otra en nuestro desarrollo, muchas de las cuales las dejamos atrás sin más, pero algunas de ellas se arraigan profundamente en nosotros, condicionando la forma que vemos el mundo. Los cambios producen pérdidas (empobrecimiento) pero también crecimiento el cual ingresa a nuestro repertorio por medio de la idea del cambio. Todo cambio implica una pérdida, y es por tanto imposible concebir el cambio sin una pérdida. Cambiamos y perdemos ante lo material, ante la pérdida de afectos, ante la más temida de las pérdidas que es la muerte y ante aquella que su materialización en cuanto pensamientos nos golpea brutalmente y que es la pérdida de aquello que nunca hemos tenido, pero si lo hemos soñado y deseado.

El manual diagnóstico de los trastornos mentales DSM-IV-TR (APA,2004), entrega una aproximación clínica al entendimiento del duelo, el cual bajo el apartado de "Problemas adicionales que pueden ser objeto de atención clínica", incluye el duelo al definirlo como la reacción ante la muerte de una persona querida.

Un gran avance en la identificación del Duelo se produce con la publicación del DSM V (APA, 2012), el cual quita la exclusión del diagnóstico Episodio Depresivo mayor, si los síntomas depresivos duraban menos de dos meses posteriores al fallecimiento de un familiar, lo cual inducía a pensar que el episodio de duelo debía durar 1 o 2 meses, 
lo que se contravenía con la observación en la práctica clínica, donde en la interacción con los consultantes, se podía identificar que estos episodios se podían arrastrar por años, sin necesariamente adscribirse en su clasificación a lo que se conoce como duelo patológico. De igual forma en el apartado "Otros problemas que pueden ser objeto de atención clínica", sitúa bajo el epígrafe V62.82 (Z63.4) el” Duelo no Complicado", asociándolo a "cuando el objeto de la atención clínica es una reacción normal ante la muerte de un ser querido". A su turno, la clasificación internacional de las enfermedades CIE 10, Trastornos Mentales y del Comportamiento, tipifica el duelo normal bajo el código Z63.4 asociando este a la desaparición o fallecimiento de un miembro de la familia), mientras que utiliza los trastornos de adaptación F43.2 para identificar las reacciones de duelo de cualquier duración que se consideren anormales por sus manifestaciones o contenidos (OMS, 1992).

\section{Algunas aproximaciones}

Sin duda que el duelo, como lo conocemos y vivimos se expresa con toda su fuerza por medio del dolor frente a la pérdida, especialmente cuando esta se origina en la pérdida de un ser querido, enfoque que tradicionalmente es objeto de estudio y análisis por diferentes autores, lo que sin embargo deja de lado los procesos de duelo que nos vemos afectados diariamente por nuestro acontecer y que nos van dejando pérdidas que muchas veces son no visibilizadas y a las cuales se le prestan tradicionalmente menos atención que a la pérdida, duelo y dolor que provoca la muerte de una persona, y que son por ejemplo término de relaciones afectivas, trabajo, estatus social y otros asociados al común devenir (Neimeyer, 2002)

La vivencia del duelo es un acto propio que se enfrenta de acuerdo a nuestras propias realidades y significación que le damos a los hechos que vivimos. De esa forma, al constituir la experiencia como un dolor propio, la significación o re significación implicará una aproximación de partida para la superación de la experiencia de dolor, del propio dolor que irrumpe e interrumpe nuestro status vivencial. El caso de Matilde (63) así al menos lo sugiere;

"La gente me decía debes ser fuerte, y yo me preguntaba ¿Por qué debo serlo? Me decían dale tiempo al tiempo, y yo no lo quería. Me decían tienes mucho por que luchar y yo no quería luchar, me decían debes de seguir viviendo y yo no quería seguir haciéndolo. Solo quería vivir este episodio a mi manera"

Matilde grita a través de sus palabras "no me quites mi duelo", déjenme vivirlo a mi manera, moviéndonos a comprender que el duelo no es un trastorno sino un proceso 
vital estresante en la mayoría de los casos por la que pasamos o pasaremos todos los seres humanos. Desde una perspectiva psicológica, el duelo provoca un desequilibrio psicoemocional que afecta todas las funciones cognitivas superiores, constituyéndose en una experiencia que nos afecta en forma global, afectando los aspectos psicológicos, emotivos, sociales, mentales, espirituales y físicos, impulsándonos a experimentar un estado de desajuste general que solo lo superaremos a través de íntimos proceso específicos de ajustes. El duelo es la búsqueda de los ajustes perdidos tras un evento de pérdidas significativas, es la búsqueda del equilibrio, es el reencuentro y aceptación de nuestro propio acontecer, de nuestra propia realidad, de nuestro propio vivenciar (Silva, 2008).

El duelo es un grito que lucha por emerger y duele. Duele la realidad, duele lo social, duelen nuestros ajustes, duele el pasado, el presente y el futuro. Duele lo hecho y lo no hecho, lo soñado y lo vivido. Toda la vida en su conjunto duele. No se debe confundir con el luto, que es la expresión física externa provocado por la pérdida, que se traduce en ritos, manifestaciones, costumbres sociales, relaciones, lo que lleva incluso a la vestimenta expresándola a través de su color negro. El duelo es una experiencia psicoemocional y el luto la expresión o manifestación externa de esa experiencia

En lo anterior subyace la expresión tradicional del duelo por la pérdida del ser querido. Sin embargo y variadas veces en la práctica de la consulta encontramos casos en que la pérdida y duelo asociado se relacionan con otros aspectos asociados al funcionamiento de la persona como un todo, por ejemplo, en el caso laboral, como lo señala Alberto:

\footnotetext{
"Cuando me lo dijeron pensé que era una broma. No podía ser que, a mí, después de estar toda una vida en la empresa, de haber postergado muchas veces mi familia y descanso me hubiesen despedido porque necesitaban reorganizar las áreas. Han pasado dos años y se fueron los amigos, la familia y los recursos. Lo único que me llegó nuevo fue esta depresión que me acompaña todos los días, como un estigma de lo que vivo". (Alberto, 59 años, Administrador)".
}

El caso de Alberto refleja una realidad que aparece conforme se transita de una economía industrial moderna a una post-moderna, donde la globalización de los mercados, la tecnología y una sociedad basada en la información, desestabilizan las empresas que se ven enfrentadas a fusiones, reorganizaciones, recortes presupuestarios y otros, haciendo añicos la seguridad y estabilidad en el empleo (Savickas, 2019). Al considerar diversos enfoques de la Psicología Laboral, las 
pérdidas laborales incluso cuando han sido planeadas, producen desorientación, períodos de incertidumbre y de reflexión en cuanto si el camino elegido fue el mejor, si la decisión fue correcta y si hubiese sido mejor no arriesgarse a un cambio. Cuando estas pérdidas se producen por otros eventos como jubilaciones forzadas, despidos u otras formas inesperadas, nos sentimos traicionados, engañados y minusvalorados, donde la pérdida del rol laboral nos producirá reacciones distintas a las producidas por pérdidas vitales, sumiendo a las personas en estados de desesperanza y desanimo, finalmente depresión, que en casos extremos puede llevar al sujeto a atentar contra su propia integridad (Ecker \& Hulley, 2008).

El trabajo, y de ahí entenderemos el impacto de la pérdida, no puede ser solo considerado una fuente de obtención de recursos monetarios, sino que define y sustenta muchos de nuestros proyectos personales, entregándonos una dirección y propósito, por el cual trabajamos y nos orientamos hacia el futuro. También organiza nuestras vidas, proporcionándonos la interacción social necesaria que nos permite relacionarnos con otras personas y mejorar nuestras capacidades en todos los frentes, en lo personal al sentir un propósito a través de él, en la interacción diaria al probar nuestras capacidades de ejecución, de resolución de problemas, de superación, de relacionarnos laboralmente y socialmente.

Algunos Psicólogos como Robert A. Neimeyer señalan que "el trabajo determina gran parte de nuestro pasado (lo que hemos conseguido), de nuestro presente (lo que hacemos cada día) y de nuestro futuro (lo que queremos conseguir), y su pérdida hacen que se tambaleen los cimientos de nuestra identidad y nuestros planes de vida". De ahí que, la pérdida del rol laboral a diferencia de otras pérdidas vitales, resultan complicadas pues no existe un período de tiempo que permita llevar un ritual que ayude a la superación, siendo necesario elaborar el duelo para superar los confusos sentimientos que la pérdida laboral provoca, y las exigencias que nos vemos enfrentados, no solo en el ámbito personal, sino que en lo familiar y social.

En la línea anterior y en la construcción de la propia identidad, el transcurso de la vida obliga a enfrentar pérdidas que no necesariamente significan el establecimiento de un duelo por medio de la muerte, sino que también por la experimentación de pérdidas relacionadas a lo afectivo, a la separación y a la carga emocional que ello conlleva, lo cual queda expresado en cruda forma en las palabras de Isabel.

"Mi mundo se derrumbó cuando Antonio me dejó. La verdad no entendía por qué después de siete años de pololeo se terminaba todo, donde hasta habíamos hablado de matrimonio. Empezamos a pololear en el Colegio y ahora en la Universidad me deja por otra. Tampoco entiendo a mis amigas 
que me dicen que ya encontraré otro, que solo le dé tiempo al tiempo. Yo lo quiero a él y solo a él. No quiero a otro en mi vida y creo que nunca volveré a creer en alguien. (Isabel, 23 años)".

La pérdida que vive Isabel no corresponde a la ausencia producida por la pérdida de un ser querido que como consecuencia final sucede la muerte, sino que obedece a lo que puede señalarse como pérdida de relaciones significativas, y que pueden hacernos entrar en ciclos emocionales que nos impidan la reorganización de nuestros significados, especialmente cuando el rompimiento de la relación es de una de las partes, que pone fin a esa relación en forma unilateral.

Como en el caso de Isabel, muchas de las pérdidas que hemos experimentado se han dado en la adolescencia, e independiente de si ellas son superficiales o no dejan profundas huellas en nosotros. La relación del pololeo a temprana edad hace percibir al otro como el centro de nuestro universo, y cuando aquello se derrumba, afloran los sentimientos de culpa, de pena, de ira, de soledad, que en algunos casos puede sumir a quien lo experimenta en una profunda depresión.

Estos sentimientos pueden verse agravados cuando los padres, amigos o círculos cercanos minimizan lo pasado, no escuchan a la parte doliente y lo toman como una situación que con el tiempo se superará. Lamentablemente, si bien en algunos casos puede ser así, en otros hace que la persona se sumerja en un profundo duelo complicado. La anterior además se ve influido porqué ninguno de nosotros entra en una relación sentimental como una página en blanco, que iremos escribiendo a medida que avanzamos, dado que más bien entramos con ciertas expectativas de como esa relación podría ser, de cómo podría evolucionar, forjando en las personas un conjunto de esperanzas y creencias, de los cuales muchas veces no somos conscientes, pero que influirán en nosotros como lo vemos en Isabel.

Una mención especial en este proceso clínico-reflexivo lo constituye la materialización del duelo no visibilizado, incomprendido, carente de atención de su círculo, el cual genera en el tiempo un mayor dolor, debido a que el dolor producido se banaliza, se trivializa, no se reconoce o se ignora, tal como a través de su brutal relato, un niño de siete años lo expresa.

"Los odio, los odio y ojalá les pase lo mismo. Mis papás y mi hermano mayor el Seba se rieron de míy me dijeron que era un tonto. Que me iban a comprar otro y que no era más que un ratón el que se había muerto. Finalmente lo tomaron y metieron en una bolsa y lo tiraron a la basura. Yo voy hacer lo mismo con ellos. Era mi hámster el que se había muerto, mi amigo que me 
miraba desde encima de la cama donde corría, con el que salía al patio a jugar y no era un ratón, tenía nombre y se llamaba Carlitos, igual que yo. No me dejaron enterrarlo como yo quería y el Seba me dijo que eran puras tonteras. Lloré y lloré y lo botaron a la basura y me castigaron mandándome a la pieza. Me trajeron otro, pero no lo quiero, ojalá se muera y se mueran ellos, los voy a meter en una bolsa de basura a todos. Carlitos (7 años)".

En el relato de Carlitos de siete años quien acude a la consulta con su mamá, "dado que se ha puesto insoportable desde que murió su mascota", empezamos a encontrar aspectos desconocidos en el proceso de la pérdida y el duelo. Cuando un familiar o alguien cercano a nosotros muere, nos permitimos entregar nuestros cariños, palabras y sinceras condolencias, entendiendo el llanto como una expresión en que el doliente y nosotros mismos estamos autorizados a realizarlo dadas las circunstancias, o sea podemos socialmente experimentar nuestras emociones (Rozalski, Holland, \& Neimeyer, 2016).

Pero que sucede cuando la pérdida es de un perro que lo atropellaron, de un gato con una enfermedad terminal, de un hámster que murió de "algo" según lo que cuenta Carlitos. En este caso la historia que se narra es distinta, y muchos te contarán que nadie y solo ellos entendieron lo que la mascota significaba, y que incluso les sugirieron comprarse otra para reemplazarla, olvidando que el duelo en esta situación es doloroso por la pérdida en sí, pero en muchos casos de una profundidad insospechada, debido a la posible soledad con que se aborda este tipo de duelo.

Cuando se pierde una mascota puede entrarse en un luto profundo por la pérdida del amor incondicional que nos entregan nuestras mascotas, por la pérdida de las emociones sinceras y por la aceptación que hacen de nosotros independiente de nuestros estados de ánimo. Ellas no nos juzgan buscando o hurgando en nuestras inseguridades o imperfecciones, aceptándonos como somos y como pocos humanos pueden lograrlo.

Estamos de luto también por la pérdida de un protegido, ya que a través de ella somos responsables - o deberíamos serlo - de otra vida, donde tenemos que hacer todo lo que está en nuestras manos para asegurarle la comodidad física y emocional de nuestros acompañantes. Somos responsables de otra vida y, a menudo hacemos todo lo posible para garantizar la comodidad física y emocional de nuestra mascota. Todos estos esfuerzos, que sin duda realizamos con cariño, nos llevan a sentir la pérdida del compañero mascota en lo más profundo de nosotros mismos. 


\section{A modo de reflexión}

En la introducción del artículo planteaba las dos visiones que se establecen como raíz de la palabra duelo, "Dolus" y "Duellum", la primera haciendo relación al dolor o aflicción que emerge ante la pérdida y el segundo, que implica un enfrentamiento entre dos personas producto de un reto o desafío, como mecanismo de superación de controversia. Así desde estas dos miradas podemos entender su complementariedad al concebir el duelo como una experiencia que intenta reconstruir el mundo de significados, enfrentado a una pérdida significativa. Las experiencias clínicas narradas nos acercan a identificar patrones de acciones donde "el doliente" debe de jugar un rol activo, más que encasillarse en etapas pre concebidas de duelo, comprometiendo su sistema de creencias.

La pérdida y la subsecuencia del duelo acercan a los pacientes a sus propias realidades y a comenzar un proceso de reconstrucción de su vivenciar, donde se busca a través de la experiencia terapéutica, centrarnos en la etapa de reconstrucción y sus significados más que enfocarse en lo que puede resultar un duelo patológico o normal, o forzar la terapia a enfocarse a tratar de lograr un estado de bienestar anterior a la pérdida. De la misma forma nos enseña el proceso terapéutico que los psicólogos debemos entender la pérdida como un proceso amplio en el cual el dolor se produce por la privación o ausencia de algo que tenemos y que no volveremos a tener, pudiendo expresarse estas de variadas formas como fue señalado en este artículo.

Así desde una mirada terapéutica, el duelo nos refleja la propia identidad del ser, nos enseña su propia construcción de significados que se ve enfrentada al momento de dolor que atraviesa el doliente, estableciendo el desafío terapéutico de mediar en la reconstrucción del mundo personal de significados que ha sido cuestionado por la pérdida. Es en este punto donde Dolus y Duellum se unen en su significado en el espacio terapéutico, al concebir el duelo como algo hecho por nosotros mismos y no impuesto desde afuera, ni menos con etapas predeterminadas como criterio de evolución universal, lo cual dejaría al doliente en un espacio pasivo desaprovechando el espacio activo que el terapeuta puede mediar. 


\section{Referencias}

American Psychiatric Association (2012). American Psychiatric Association. DSMV Development: Persistent Complex Bereavement- Related Disorder (Proposed for Section III of the DSM-5).

American Psychiatric Association (2004). Asociación Psiquiátrica Americana (APA). Manual Diagnóstico y Estadístico de los Trastornos Mentales, Texto Revisado. DSM IVTR. Masson.

Barreto, MP, Soler C. (2007). Muerte y duelo. Síntesis.

Bowlby, J. (1993). La pérdida afectiva: tristeza y depresión. Paidós

Ecker, B. \& Hulley, L. (2008). Coherence Therapy: Swift change at the roots of symptom production. In J. Raskin \& S. Bridges (Eds.), Studies in meaning 3: Constructivist psychotherapy in the real world. Pace University Press.

Meza, E. ; García, S.; Torres, A.; Castillo, L.; Sauri, S. \& Martíne, S. (2008). El proceso del duelo. Un mecanismo humano para el manejo de las pérdidas emocionales, Revista de Especialidades Médico-Quirúrgicas, 13,1.

Neimeyer, R. (2002). Lessons of loss: a guide to coping. Memphis, TN: Center for the Study of Loss and Transition.

Organización Mundial de la Salud (1992). Organización Mundial de la Salud, CIE. Clasificación internacional de las enfermedades: Trastornos mentales y del comportamiento. $10^{\mathrm{a}}$ ed. Meditor.

Real Academia Española (2001). Real Academia de la Lengua Española. 22 ed. Espasa, Apraxia: 854.

Rozalski, V., Holland, J. M. \& Neimeyer, R. A. (2016). Circumstances of death and complicated grief: Indirect associations through meaning made of loss. Journal of Loss and Trauma, 10.1080/15325024.2016.1161426.

Savickas, M. (2019). Career Counseling. American Psychological Association. 2ed.

Silva, B (2008). El proceso del duelo. Un mecanismo humano para el manejo de las pérdidas emocionales. Revista de Especialidades Médico-Quirúrgicas, 13,1. 


\section{Ricardo L. Guzmán Sanza}

Psicólogo, UNIACC, Ingeniero Comercial, Universidad Arturo Prat, Profesor de Estado en Física, Universidad de Chile, Doctor of Business Administratión - DBA, ULSETB, Bruxelles, Bélgica; Revalidado Doctor en Administración de Empresas - Universidad de Chile, Master en Dirección de Empresas - MBA, Universidad de Lleida, España, Master of Business Administration; P. Universidad Católica, Magíster en Administración de Empresas, P. Universidad Católica, Magíster en Ciencias Políticas, Universidad de Chile, Post- titulado en Estudios Superiores en Administración de Empresas (ESAE), P. Universidad Católica. Profesor de Administración y Gestión de Recursos Humanos, Diseño y cambio Organizacional, Comportamiento y Desarrollo Organizacional y Negociación Internacional en Universidad del Pacífico y Profesor de Fundamentos Estadísticos y Metodología Cuantitativa, UNIACC. E.mail: ricardo.guzman@uniacc.edu 